\title{
Vortices in a Bose-Einstein condensate confined by an optical lattice
}

\author{
P G Kevrekidis ${ }^{1}$, R Carretero-González ${ }^{2}$, G Theocharis ${ }^{3}$, \\ D J Frantzeskakis ${ }^{3}$ and B A Malomed ${ }^{4}$ \\ ${ }^{1}$ Department of Mathematics and Statistics, University of Massachusetts, Amherst, \\ MA 01003-4515, USA \\ 2 Nonlinear Dynamical Systems Group ${ }^{5}$, Department of Mathematics and Statistics, \\ San Diego State University, San Diego, CA 92182-7720, USA \\ ${ }^{3}$ Department of Physics, University of Athens, Panepistimiopolis, Zografos, Athens 15784, \\ Greece \\ ${ }^{4}$ Department of Interdisciplinary Studies, Faculty of Engineering, Tel Aviv University, \\ Tel Aviv 69978, Israel
}

Received 2 April 2003, in final form 16 June 2003

Published 5 August 2003

Online at stacks.iop.org/JPhysB/36/3467

\begin{abstract}
We investigate the dynamics of vortices in repulsive Bose-Einstein condensates in the presence of an optical lattice (OL) and a parabolic magnetic trap. The dynamics is sensitive to the phase of the OL potential relative to the magnetic trap, and depends less on the OL strength. For the cosinusoidal OL potential, a local minimum is generated at the trap's centre, creating a stable equilibrium for the vortex, while in the case of the sinusoidal potential, the vortex is expelled from the centre, demonstrating spiral motion. Cases where the vortex is created far from the trap's centre are also studied, revealing slow outward-spiralling drift. Numerical results are explained in an analytical form by means of a variational approximation. Finally, motivated by a discrete model (which is tantamount to the case of the strong OL lattice), we present a novel type of vortex consisting of two pairs of antiphase solitons.
\end{abstract}

(Some figures in this article are in colour only in the electronic version)

The experimental realization and theoretical studies of Bose-Einstein condensates (BECs) [1] have led to an explosion of interest in the field of atomic matter waves and their nonlinear excitations, including dark [2] and bright [3] solitons. More recently, two-dimensional (2D) excitations, such as vortices [4] and vortex lattices [5], were considered and realized experimentally. Other nonlinear states, such as for example Faraday waves [6], ring dark solitons and vortex necklaces [7], stable solitons and localized vortices in attractive BECs

5 http://nlds.sdsu.edu/ 
trapped in a 2D optical lattice (OL) [8] and even stable solitons supported by an OL in a repulsive $\mathrm{BEC}[9]$, have also been predicted.

Vortices, in particular, are worth studying not only because of their significance as a fundamental type of coherent nonlinear excitation, but also because they play a dominant role in the breakdown of superflow in Bose fluids $[10,11]$. The theoretical description of vortices in BECs can be carried out in a much more efficient way than in liquid He [12] due to the weakness of interactions in the former case (which, in addition, are tunable [13]). These advantages explain a large volume of work regarding the behaviour of vortices in BECs, which has been recently summarized in a review by Fetter and Svidzinsky [14].

The subject of the present paper is the dynamics of vortices under the action of the OL; to the best of our knowledge, this problem is considered in this work for the first time. An OL potential is generated as an interference pattern by laser beams illuminating the condensate, in particular in $1 \mathrm{D}$ and 2D cases [15-19]. In the 2D case, the OL potential assumes the form (in dimensionless units)

$$
V_{\mathrm{OL}}(x, y)=V_{0}\left[\cos ^{2}(k x+\phi)+\cos ^{2}(k y+\phi)\right],
$$

where $V_{0}$ is the strength of the OL, which is measured in units of the recoil energy $E_{\mathrm{r}}=h^{2} / 2 m \lambda_{\text {laser }}^{2}$ (i.e. the kinetic energy gained by an atom when it absorbs a photon from the OL), where $\lambda_{\text {laser }}$ is the laser wavelength, $h$ is Planck's constant, $m$ is the atomic mass, $k$ is the wavenumber of the OL, and $\phi$ is a phase-detuning factor (the obvious possibility to remove $\phi$ by means of the diagonal shift, $(x, y) \rightarrow(x-\phi / k, y-\phi / k)$, is ruled out by the presence of the magnetic-trap potential; see below). The wavenumber $k$ of the OL can be experimentally controlled by varying the angle between the counter-propagating lasers producing the interference pattern [20].

As is well known, the effective 2D GP equation applies to situations when the condensate has a nearly planar ('pancake') shape (see, e.g., [21] and references therein). Accordingly, vortex states considered below are not subject to 3D instabilities (corrugation of the vortex axis [14]) as the transverse dimension is effectively suppressed. We base the analysis on the Gross-Pitaevskii (GP) equation, written in harmonic-oscillator units [22],

$$
\mathrm{i} u_{t}=-\Delta u+|u|^{2} u+V(x, y) u,
$$

where $u=u(x, y, t)$ is the $2 \mathrm{D}$ wavefunction, and the external potential is

$$
V(x, y)=\frac{1}{2} \Omega^{2}\left(x^{2}+y^{2}\right)+V_{\mathrm{OL}}(x, y),
$$

which includes the isotropic magnetic trap [1] and OL potential (1). Notice that the dimensionless parameter $\Omega \equiv \omega_{\mathrm{r}} / \omega_{\mathrm{z}}$ in equation (3), where $\omega_{\mathrm{r}, \mathrm{z}}$ are the confinement frequencies in the radial and axial directions respectively, is assumed to be $\Omega \ll 1$. In most studies of vortices, angular momentum imparted by stirring of the condensate is assumed (see, e.g., $[23,24]$ and references therein), as typically this is the way in which vortices are generated in experimental settings [4]. Here we assume that a vortex has been generated, but then the stirring ceases. Alternatively, the vortex may be created by means of the phase-engineering technique [25]. The positive sign in front of the nonlinear term in equation (2) implies that we consider a repulsive condensate.

We first present numerical findings for the case of a 'regular' vortex; subsequently, the observed dynamics is explained by means of a variational approximation. We then proceed to examine a new type of a vortex, which is a robust bound state of two pairs of $\pi$-out-ofphase pulses, as suggested by results known for 2D dynamical lattices. In most cases, we fix the strength of the magnetic-trap potential to be $\Omega^{2}=0.002$. This value is relevant to a ${ }^{87} \mathrm{Rb}$ condensate of radius $25 \mu \mathrm{m}$, containing $4.6 \times 10^{4}$ atoms in a highly anisotropic trap with $\omega_{\mathrm{r}}=2 \pi \times 7.5 \mathrm{~Hz}$ and $\omega_{\mathrm{z}}=2 \pi \times 115 \mathrm{~Hz}$. According to these values of the physical 

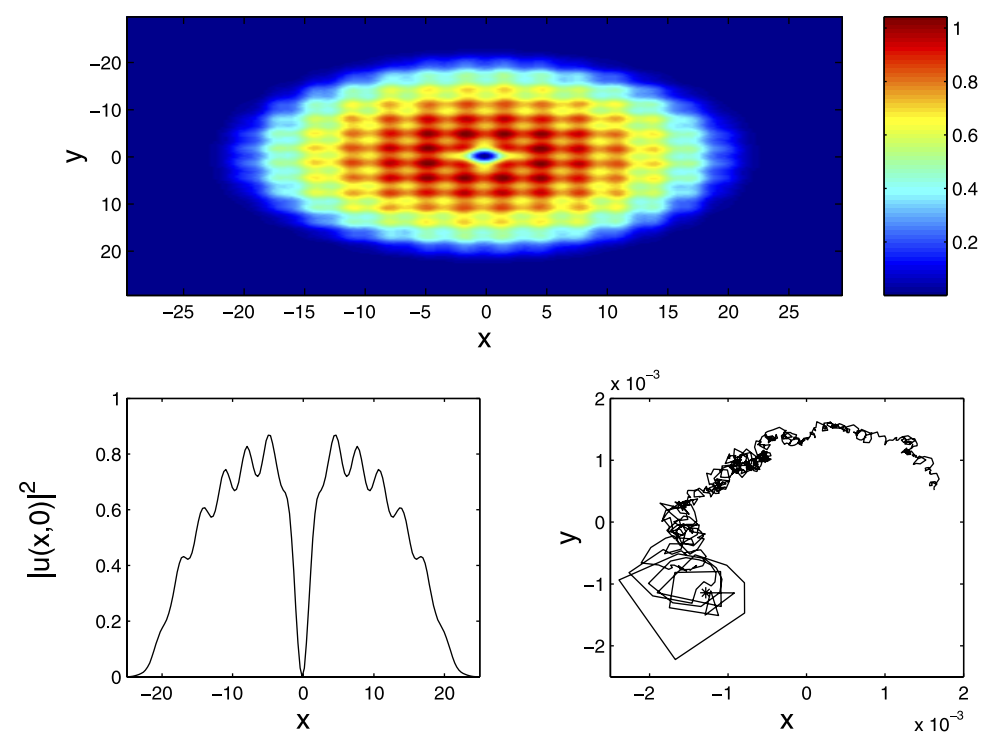

Figure 1. The vortex on a BEC of diameter $\approx 50 \mu \mathrm{m}$ in the cosinusoidal $(\phi=0$ in equation $(1))$ OL potential with $V_{0}=0.5$ and $k=1$. The top panel shows the contour plot of the density at $t=100(138 \mathrm{~ms})$. The bottom left panel is a cut of the same density profile along $x=0$, while the bottom right one shows the motion of the vortex centre for $0 \leqslant t \leqslant 100$, the initial position being marked by a star. Notice the scale $\left(10^{-3}\right.$, or $1 \mathrm{~nm}$ in physical units) of very weak jiggling of the vortex, which thus stays practically immobile at the origin.

parameters, in the following results, which will be presented in normalized time and space units, the corresponding units are $1.38 \mathrm{~ms}$ and $1 \mu \mathrm{m}$ respectively. Additionally, as far as the OL parameters are concerned, the typical values $V_{0}=0.5$ and $k=1$ used in most cases, correspond to $0.5 E_{\mathrm{r}}$ and to an OL wavelength of $6.3 \mu \mathrm{m}$.

In simulations, the initial vortex configuration is taken, in polar coordinates $\rho$ and $\theta$, as

$$
u=\rho(r) \exp (\mathrm{i} \theta) u_{\mathrm{TF}},
$$

where $\rho^{2}(r)=r^{2}\left(0.34+0.07 r^{2}\right) /\left(1+0.41 r^{2}+0.07 r^{4}\right)$ is the radial Padé interpolation for the vortex solution to the GP equation without external potential, and $u_{\mathrm{TF}}=$ $\sqrt{\max \left\{0, \mu-\left(\Omega^{2} / 2\right)\left(x^{2}+y^{2}\right)\right\}}$ is the Thomas-Fermi (TF) wavefunction for the magnetic trap [1], with a chemical potential $\mu$. The vortex is placed at the centre of the magnetic trap (unless otherwise indicated). Simulations were performed by means of a finite-difference discretization in a box of the size $60 \times 60(60 \mu \mathrm{m} \times 60 \mu \mathrm{m})$, using $200 \times 200$ points (i.e. the spatial step sizes are $\mathrm{d} x=\mathrm{d} y=0.3)$. Time integration was performed by means of the fourth-order Runge-Kutta scheme with the time step $\mathrm{d} t=0.0025(\mathrm{~d} t=3.45 \mu \mathrm{s})$. It should be remarked that in the initial stages of the time evolution, the initial condition given by equation (4) 'adjusts' itself to the OL, by shedding small amplitude radiation waves. These are dissipated by the an absorbing region close to the boundary implemented in the numerical simulation.

The first case that was examined is the one with $\phi=0$ in equation (1). In this case, the vortex is extremely robust, staying at the centre during a few hundred time units of equation (2), which correspond to a few hundred milliseconds in physical units. As can be seen in figure 1, where the vortex and its motion are shown for $V_{0}=0.5$ and $k=1$, the displacement of the vortex centre remains, for an indefinitely long time, as small as $\sim 10^{-3}$. The centre of the vortex was located by fitting, close to the local minimum, the density $|u|^{2}$ to the expression $|u|^{2}=A x^{2}+B y^{2}+C x+D y+E$, which yields the position of the centre at $(-C / 2 A,-B / 2 D)$. 

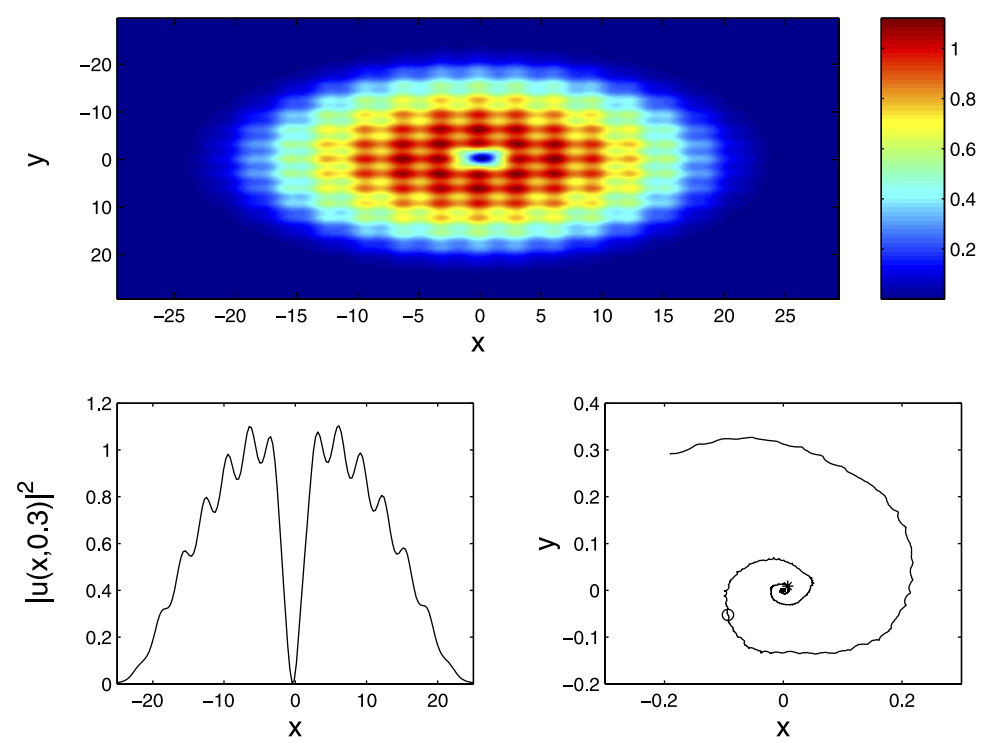

Figure 2. The same as in figure 1 , but now the potential is sinusoidal, $\phi=\pi / 2$. To clearly demonstrate the dynamics, the top and bottom left panels show the density profiles at a larger time than in figure $1, t=250$ (346 ms). The bottom right panel depicts the motion of the vortex centre for $0 \leqslant t \leqslant 250$ (positions of the vortex centre at $t=100(138 \mathrm{~ms}$ ) and $t=200(276 \mathrm{~ms})$, respectively, are indicated by the asterisk and circle).

In figure 2, we display the evolution which starts from the same initial configuration, but with $\phi=\pi / 2$ in equation (1). A distinctly different behaviour is observed in this case, which is shown for longer times, up to $t=250(346 \mathrm{~ms})$, to verify that it is a real feature. In particular, it is clearly observed in this case that the vortex spirals out from the centre of the magnetic trap. Note that fine details of the motion indicate meandering motion, similar to that exhibited by spiral waves in excitable media [26], even though in the present case the meandering occurs on a smaller spatial scale, hence much finer resolution would be needed to clarify it. However, the outward-spiralling motion of the vortex centre is clearly seen in figure 2 . Considerably longer runs (for times of the order of a few thousand time units of equation (2), which correspond to seconds in physical units) show that the vortex spirals all the way to the edges of the TF cloud where it eventually decays (see also comments below).

In figure 3 we examine the case with $\phi=\pi / 4$ in equation (1). In this case also, the vortex moves outward. In fact, it is observed that it moves away from the trap centre much faster, as at $t=100(138 \mathrm{~ms})$ it is already found near $(x, y)=(1.5,-1.5)$ (cf figure 2$)$, corresponding to a couple of micrometres from the trap centre.

Qualitatively similar conclusions, but at different time scales, have been obtained for different values of the OL strength $V_{0}$. For instance, in the potential with $\phi=\pi / 2, V_{0}=1.5$ (and $k=1$ ), the vortex spirals out again (similar to the case with $V_{0}=0.5$ displayed above), the time necessary for it to reach the distance of 0.1 from the origin being three times as large as in the case of $V_{0}=0.5$ (details are not shown here). This can be explained by the fact that if $V_{0}$ is larger the vortex has to move through a 'rougher' energy landscape, hence it becomes more difficult for it to 'find its way out'.

These findings can be qualitatively understood in terms of an effective potential which governs the motion of the vortex; in particular, the potential has a minimum and maximum at the centre of the magnetic trap in the cases of $\phi=0$ and $\pi / 2$ respectively. To present this 

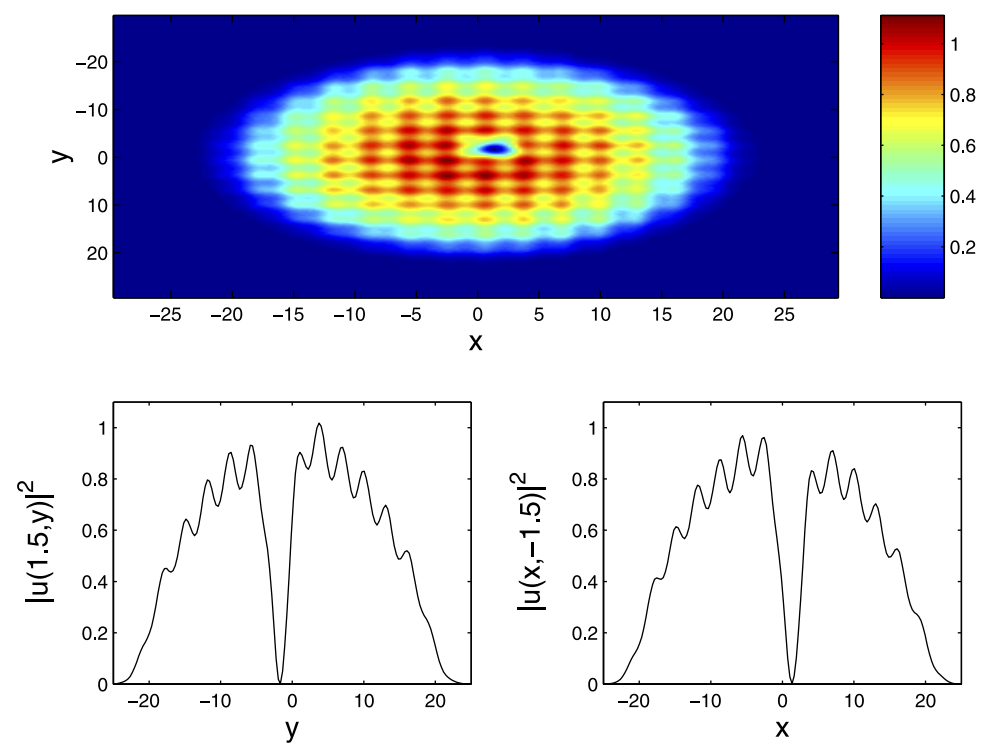

Figure 3. The case of $V_{0}=0.5$ and $\phi=\pi / 4$ in equation (1). The vortex moves rapidly away from the centre. The snapshots show the density profile (top panel) and its horizontal and vertical cuts (along $x=1.5$ and $y=-1.5$, respectively) of the field at $t=100$ (138 ms).

explanation in a mathematically tractable form, we resort to a variational approximation. As in [27], we use the following ansatz for the vortex, inspired by its linear analogue namely the first excited state of the quantum harmonic oscillator:

$$
u(x, y, t)=B(t) r_{0}(t) \exp \left[-r_{0}^{2}(t) / b(t)\right] \mathrm{e}^{\mathrm{i} \varphi_{0}(t)},
$$

where $r_{0}^{2}(t) \equiv\left(x-x_{0}(t)\right)^{2}+\left(y-y_{0}(t)\right)^{2}$, and $\varphi_{0}(t) \equiv \tan ^{-1}\left[\left(y-y_{0}(t)\right) /\left(x-x_{0}(t)\right)\right]$. Note that the ansatz carries a vortex-like structure centred at $\left(x_{0}, y_{0}\right)$, and it qualitatively emulates the initial waveform (4) which was adopted in the numerical simulations. The stronger the nonlinearity, the less accurate the approximation offered by equation (5) is; however, as the ansatz (5) was found to always provide a qualitatively correct description of the phenomenology, we use it to represent the vortex.

Similarly to the calculations reported in [28,29], one can easily deduce (additionally using the norm conservation) that the evolution of $B(t)$ and $b(t)$ is, to the leading order, negligible, therefore we set them to constant values, $B(t) \approx B(0)$ and $b(t) \approx b(0)$. Then, the substitution of the ansatz (5) into the Lagrangian of the GP equation (2) with the potential (3) leads, up to constant factors, to the effective Lagrangian

$$
L=\frac{1}{2}\left(\dot{x}_{0}^{2}+\dot{y}_{0}^{2}\right)-V_{\text {eff }}\left(x_{0}, y_{0}\right),
$$

where the net effect of the OL and magnetic trap is combined into an effective potential,

$$
V_{\mathrm{eff}}(x, y)=Q(\phi)[\cos (2 k x)+\cos (2 k y)]+\frac{1}{4} \Omega^{2}\left(x^{2}+y^{2}\right),
$$

where $Q(\phi)$ is given by a rather cumbersome expression; we will actually need the values

$$
\begin{aligned}
& Q(0)=\frac{V_{0}}{8}\left(b k^{2}-2\right) \exp \left(-b k^{2} / 2\right) \\
& Q\left(\frac{\pi}{2}\right)=-Q(0) \quad \text { and } \quad Q\left(\frac{\pi}{4}\right)=0 .
\end{aligned}
$$



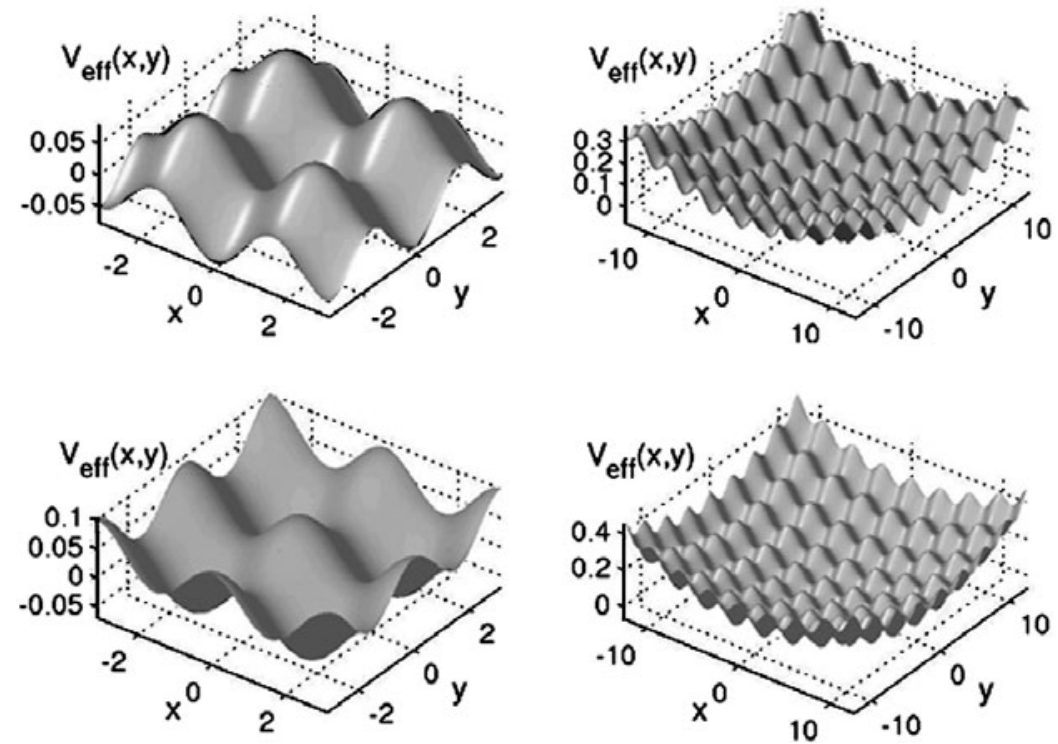

Figure 4. The effective potential $V_{\text {eff }}(x, y)$, obtained in the variational approximation (cf equation (7)), responsible for the motion of the vortex subject to the 2D OL and magnetic trap. Top panels: the cosinusoidal OL $(\phi=0)$ produces a minimum in the effective potential at the origin. Bottom: the sinusoidal optical potential $(\phi=\pi / 2)$ gives rise to a maximum of the potential at the origin. The right panels display more clearly the combined effect of the OL and magnetic trap (here we use $\Omega^{2}=0.005$ instead of $\Omega^{2}=0.002$ which was used in the computations). The parameters are $b=1, k=1, V_{0}=0.5$.

Equations (6) and (7) suggest that the motion of the coordinates $x_{0}$ and $y_{0}$ of the vortex core resembles the motion of two uncoupled oscillators. Actually, this is a straightforward generalization of a result that can be obtained in the corresponding 1D case: in that case, the counterpart of the vortex is a dark soliton, whose equation of motion in the presence of the potential (3) can be derived by means of the adiabatic perturbation theory for dark solitons in BECs (see, e.g., [30] and references therein). This approach, in the presence of the onedimensional magnetic trap and OL, yields an effective potential

$$
V_{\mathrm{eff}}^{(1 \mathrm{D})}(x)=\frac{1}{4} V_{0}\left[1-\frac{1}{6}\left(\frac{\pi^{2}}{3}-2\right) k^{2}\right] \cos (2 k x)+\frac{1}{4} \Omega^{2} x^{2} .
$$

In fact, equation (7) is a generalization of this expression. The effective potential from equation (7) is depicted in figure 4 as a function of $x$ and $y$ for the cases $\phi=0$ and $\pi / 2$, where we set $b=1$ (this value was chosen, comparing the size of the numerically obtained vortex with that implied by the ansatz (5)).

In our experiments $k=1$ was used, and thus, in the case $\phi=0$, a vortex positioned at the centre of the magnetic trap is at a local minimum of the potential (7), therefore the vortex stays in this position. If slightly perturbed around this stable fixed point, the vortex will perform small-amplitude oscillations. On the contrary, in the case of $\phi=\pi / 2$, the same point $\left(x_{0}, y_{0}\right)=(0,0)$ is unstable (as a saddle), and as a result the vortex moves away from it. These conclusions are in full accord with the numerical experiments (see figures 1 and 2).

Normally, in a Hamiltonian mechanical system the motion from an unstable saddle point occurs along the corresponding unstable separatrix. However, it is well known [31-33] that a spatially periodic setting may give rise to resonant effects, which induce an effective dissipation, 

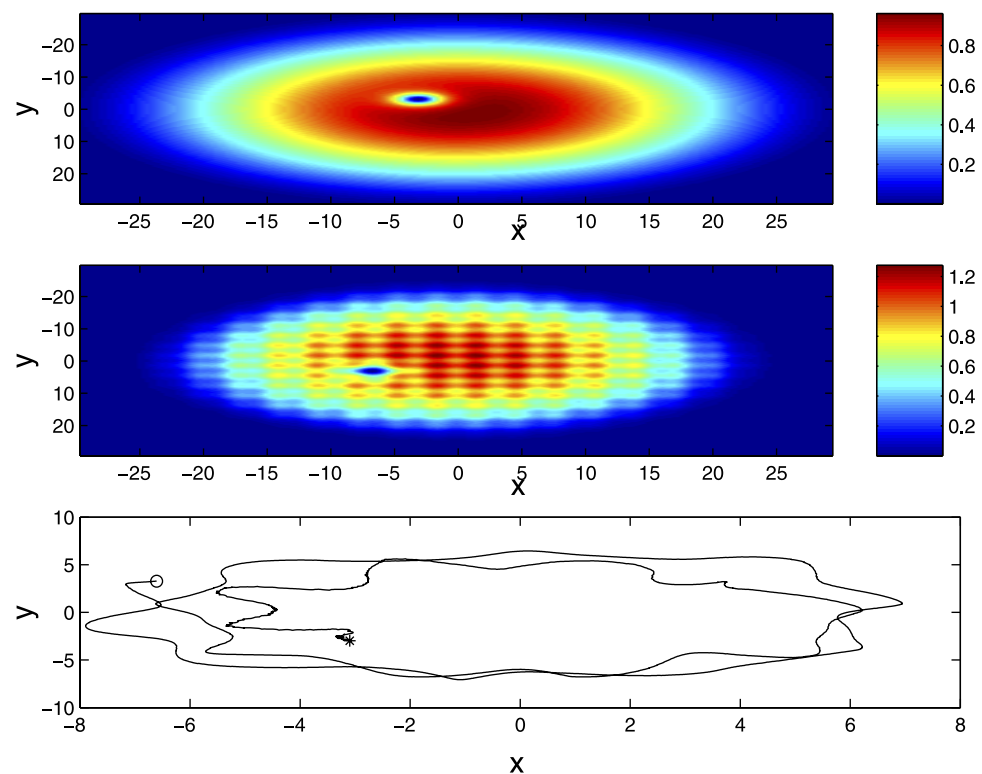

Figure 5. The top and middle panels show the vortex configuration at $t=0$ and $750(\approx 1 \mathrm{~s})$. The bottom panel shows the trajectory of the vortex from $t=0$ up to 750 (the two positions are marked by the asterisk and circle respectively).

due to emission of radiation waves from a nonlinear-wave state (vortex, in the present case). This effective dissipation, as is known [24, 34-36], causes the vortex to spiral outward (making the saddle point look like an unstable spiral), which is what we indeed observe in the simulations (see figure 2). Note that in other cases where the Hamiltonian is not positive definite (such as, e.g., Korteweg-de Vries-type models), radiative losses may destabilize an equilibrium position which is otherwise stable [37]. Finally, in the case of $\phi=\pi / 4$, the centre of the vortex is not originally at an equilibrium position of the potential of equation (1), hence its drift is manifested faster, as is indeed seen in figure 3.

Now we turn to a different case, in which the vortex was created far from the centre of the magnetic trap (the results are displayed only for $\phi=0$, as it was found that the value of $\phi$ does not significantly affect the results in this case). The motion of the vortex is displayed in figure 5, where its outward-spiralling, due to the effective dissipation induced by the OL, is obvious. This spiral motion is combined with jiggling induced by the potential energy landscape. Thus, we conclude that a vortex seeded in the periphery of the BEC cloud will slowly drift towards the edge of the cloud, where it will eventually decay into other excitations [35].

Finally, we present a novel type of vortex, which is quite different from the one considered above. Motivated by recent investigations of vortices on 2D discrete lattices [38, 39], we initialized a real field configuration which is shown in the top panel of figure 6 . The configuration consists of two up-down (dipolar) pulse pairs, each featuring a phase shift of $\pi$, hence a total phase shift along a contour surrounding these pulses is $2 \pi$, which corresponds to unit vorticity. In discrete lattices, numerically exact stable stationary solutions of this type exist [38]. In spatially uniform continuum models, such stationary solutions cannot exist, but figure 6 shows that they may be sustained by the OL. After shedding some radiation, the configuration reaches a nearly steady state, although residual oscillations are observed. Notice, once again, that waves radiated away due to the oscillations are absorbed by the dissipative 

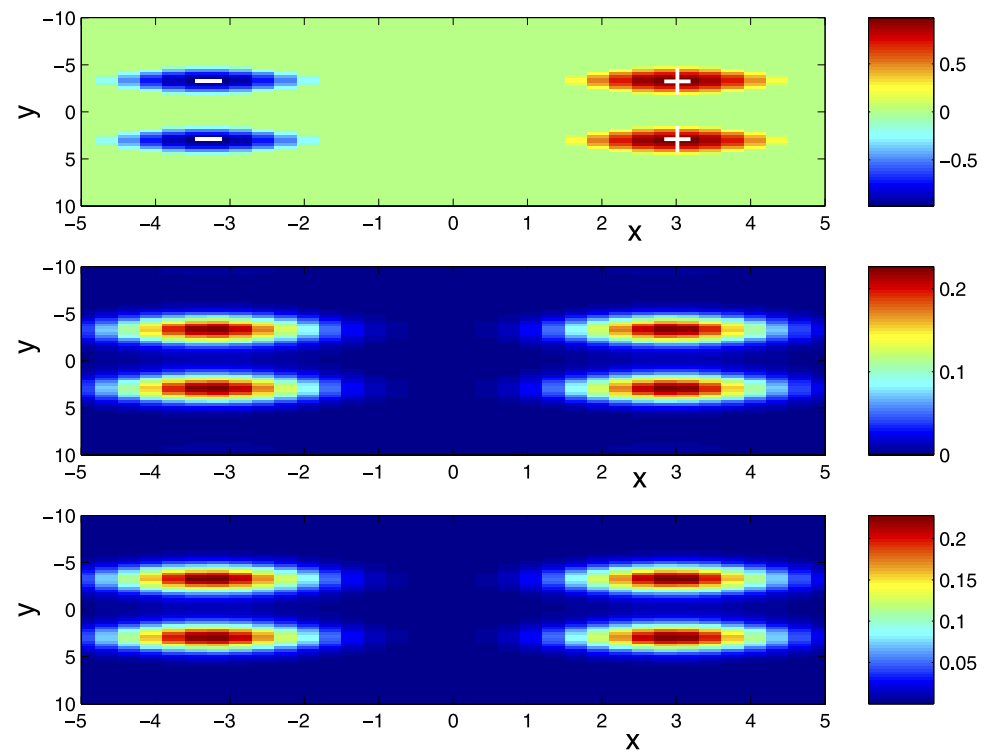

Figure 6. The top panel shows the contour plot of the real part of the initial configuration consisting of two pairs of pulses which are $\pi$ out of phase (hence the total phase change, as one goes around the configuration is $2 \pi$ ). The pluses indicate (centres of) the 'up-pulses', whereas the minus signs show the 'down-pulses' of the initial configuration. The middle and bottom panel show the contour plot of the square modulus of the wavefunction at $t=100$ and 200 respectively. In the latter, we have verified that the total phase shift around the pulse quartet is $2 \pi$, hence the vortex retains its topological charge. In this case, $V_{0}=2, k=0.5, \phi=0$, and $\Omega=0$.

boundaries of the integration domain (which were imposed to avoid artificial reflection). This dynamically stable, lattice-motivated configuration represents, to the best of our knowledge, a novel vortex-like structure which is particular to the system equipped with OL: without the lattice potential, the two $\pi$-out-of-phase sets of the in-phase pulses would separate due the pulse-pulse interactions (which are repulsive in the case of opposite 'parity' pulses and attractive in the case of same parity ones; see, e.g., [40]). However, the effective potential exerts a 'local force' on the pulses, making it possible to trap them together in a stable configuration of this type.

In conclusion, we have studied basic properties of vortices in the 2D GP equation with the OL and magnetic trap. The results crucially depend on the phase of the OL relative to the parabolic magnetic trap. Depending on the phase, it is possible to trap the vortex at the centre of the trap, or, on the contrary, to expel it, which is readily explained in terms of an effective potential for the vortex derived by means of the variational approximation. In the latter case, the vortex moves along an unwinding spiral, which is explained by the effective dissipation due to emission of small-amplitude waves. We also considered the case in which the vortex was initially created far from the centre of the trap, for which a precessing motion with a slow outward-spiralling drift was observed and qualitatively explained. Finally, a new species of a stable vortex, endemic to the system equipped with the OL, was found in the form of a bound state of two $\pi$-out-of-phase soliton pairs. The existence of this vortex is also explained by the OL-induced potential.

Naturally, it would be of interest to further examine structures which are inspired by features particular to periodically modulated models, and to observe how they appear and disappear with variation of the OL strength. Such studies are currently in progress. 


\section{Acknowledgments}

This work was supported by a UMass FRG and NSF-DMS-0204585 (PGK), the Special Research Account of the University of Athens (GT, DJF), the Binational (US-Israel) Science Foundation, under grant no 1999459 (BAM), and San Diego State University Foundation (RCG).

\section{References}

[1] Dalfovo F, Giorgini S, Pitaevskii L P and Stringari S 1999 Rev. Mod. Phys. 71463

[2] Burger S et al 1999 Phys. Rev. Lett. 835198 Denschlag J et al 2000 Science $\mathbf{2 8 7} 97$ Anderson B P et al 2001 Phys. Rev. Lett. 862926

[3] Strecker K E et al 2002 Nature 417150 Khaykovich L et al 2002 Science 2961290

[4] Matthews M R et al 1999 Phys. Rev. Lett. 832498 Madison K W et al 2000 Phys. Rev. Lett. 84806 Inouye S et al 2001 Phys. Rev. Lett. 87080402

[5] Abo-Shaeer J R et al 2001 Science 292476 Abo-Shaeer J R, Raman C and Ketterle W 2002 Phys. Rev. Lett. 88070409 Engels P et al 2002 Phys. Rev. Lett. 89100403

[6] Staliunas K, Longhi S and de Valcárcel G J 2002 Phys. Rev. Lett. 89210406

[7] Theocharis G, Frantzeskakis D J, Kevrekidis P G, Malomed B A and Kivshar Yu S 2003 Phys. Rev. Lett. 90 120403

[8] Baizakov B B, Malomed B A and Salerno M 2003 Multidimensional solitons in periodic potentials Europhys. Lett. at press (Preprint cond-mat/0306645)Preprint

[9] Baizakov B B, Konotop V V and Salerno M 2002 J. Phys. B: At. Mol. Opt. Phys. 355105

[10] Frisch T, Pomeau Y and Rica S 1992 Phys. Rev. Lett. 691644

[11] Winiecki T, McCann J F and Adams C S 1999 Phys. Rev. Lett. 825186

[12] Donnelly R J 1991 Quantized Vortices in He II (Cambridge: Cambridge University Press)

[13] Inouye S et al 1998 Nature 392151 Donley E A et al 2001 Nature 412295

[14] Fetter A L and Svidzinsky A A 2001 J. Phys.: Condens. Matter 13 R135

[15] Greiner M, Block I, Mandel O, Hänsch T W and Esslinger T 2001 Appl. Phys. B 73769

[16] Trombettoni A and Smerzi A 2001 Phys. Rev. Lett. 862353

[17] Abdullaev F Kh, Baizakov B B, Darmanyan S A, Konotop V V and Salerno M 2001 Phys. Rev. A 64043606

[18] Smerzi A, Trombettoni A, Kevrekidis P G and Bishop A R 2002 Phys. Rev. Lett. 89170402

[19] Cataliotti F S et al 2002 Preprint cond-mat/0207139

[20] Morsch O and Arimondo E 2002 Dynamics and Thermodynamics of Systems with Long-Range Interactions ed T Dauxois, S Ruffo, E Arimondo and M Wilkens (Berlin: Springer) pp 312-31

[21] Band Y B, Towers I and Malomed B A 2003 Phys. Rev. A 67023602

[22] Ruprecht P A, Holland M J, Burnett K and Edwards M 1995 Phys. Rev. A 514704

[23] García-Ripoll J J and Pérez-García V M 2001 Phys. Rev. A 63041603 (Preprint cond-mat/0012071)

[24] Jackson B, McCann J F and Adams C S 1999 Phys. Rev. A 61013604

[25] Williams J E and Holland M J 1999 Nature 401568

[26] Sandstede B and Scheel A 2001 Phys. Rev. Lett. 86171

[27] Tempere J and Devreese J T 2002 Physica C 36928

[28] Carretero-González R and Promislow K 2002 Phys. Rev. A 66033610

[29] Carretero-González R and Promislow K Breathing behaviour of coupled Bose-Einstein condensates: from multi-soliton interactions to homoclinic tangles Preprint see: http://www.rohan.sdsu.edu/ rcarrete/publications/

[30] Frantzeskakis D J, Theocharis G, Diakonos F K, Schmelcher P and Kivshar Yu S 2002 Phys. Rev. A 66053608

[31] Peyrard M and Kruskal M D 1984 Physica D 14 88-102

[32] Kivshar Yu S and Malomed B A 1989 Rev. Mod. Phys. 61763

[33] Kevrekidis P G and Weinstein M I 2000 Physica D 142113

[34] Rokshar D S 1997 Phys. Rev. Lett. 792164

[35] Fedichev P O and Shlyapnikov G V 1999 Phys. Rev. A 60 R1779 
[36] Hess G B 1967 Phys. Rev. 161189

[37] Malomed B A 1988 Physica D 32393

[38] Malomed B A and Kevrekidis P G 2001 Phys. Rev. E 64026601

[39] Kevrekidis P G, Malomed B A and Gaididei Yu B 2002 Phys. Rev. E 66016609

[40] Kapitula T, Kevrekidis P G and Malomed B 2001 Phys. Rev. E 63036604 